\title{
Thiazolidinediones and Advanced Liver Fibrosis in Nonalcoholic Steatohepatitis A Meta-analysis
}

Giovanni Musso, MD; Maurizio Cassader, PhD; Elena Paschetta, MD; Roberto Gambino, PhD

IMPORTANCE Nonalcoholic steatohepatitis (NASH) is projected to be the leading cause of liver transplantation by 2020. Advanced fibrosis (stage F3-F4) on liver biopsy independently predicts all-cause and liver-related mortality in NASH. There are no known efficacious treatments for advanced fibrosis related to NASH. Thiazolidinedione therapy has been extensively evaluated in NASH, and new randomized clinical trials (RCTs) of its efficacy have been completed.

OBJECTIVE To synthesize the evidence about the association of thiazolidinedione therapy with advanced liver fibrosis in NASH.

DATA SOURCES MEDLINE, Ovid MEDLINE In-Process, Cochrane Library, EMBASE, clinicaltrials.gov, PubMed, and Scopus databases (without language restrictions), as well as other registries and scientific meeting presentations, from database inception through August 15, 2016.

STUDY SELECTION Randomized clinical trials evaluating the effect of thiazolidinedione therapy on histologic features of the liver in biopsy-proven NASH.

DATA EXTRACTION AND SYNTHESIS Two investigators extracted study data independently and in duplicate and rated the risk of bias using the Cochrane Risk of Bias Tool.

MAIN OUTCOMES AND MEASURES The primary outcome was a dichotomous improvement in advanced fibrosis on liver biopsy, defined as an improvement in fibrosis stage from F3-F4 to FO-F2. Secondary outcomes were at least a 1-point improvement in fibrosis of any stage and NASH resolution. This meta-analysis also evaluated adverse effects of thiazolidinedione therapy, including weight gain, lower limb edema, congestive heart failure, bone fractures, cancer, and anemia. With the use of random-effects models, dichotomous variables are presented as odds ratios (ORs) with $95 \% \mathrm{Cls}$, and continuous variables are presented as weighted mean differences with $95 \% \mathrm{Cls}$.

RESULTS This study analyzed 8 RCTs ( 5 evaluating pioglitazone use and 3 evaluating rosiglitazone maleate use) enrolling 516 patients with biopsy-proven NASH for a duration of 6 to 24 months. Among all studies combined, thiazolidinedione therapy was associated with improved advanced fibrosis (OR, 3.15; 95\% Cl, 1.25-7.93; $P=.01 ; I^{2}=0 \%$ ), fibrosis of any stage (OR, 1.66; $\left.95 \% \mathrm{Cl}, 1.12-2.47 ; P=.01 ; P^{2}=0 \%\right)$, and NASH resolution $(\mathrm{OR}, 3.22 ; 95 \% \mathrm{Cl}$, 2.17-4.79; $P<.001 ; P^{2}=0 \%$ ). Analyses restricted to RCTs enrolling patients without diabetes yielded similar results for improvement in advanced fibrosis (OR, 2.95; 95\% Cl, 1.04-10.90; $\left.P=.02 ; l^{2}=0 \%\right)$, improvement in fibrosis of any stage $(\mathrm{OR}, 1.76 ; 95 \% \mathrm{Cl}, 1.02-3.03 ; P=.02$; $P^{2}=0 \%$ ), and NASH resolution (OR, 3.40; 95\% Cl, 1.95-5.93; $P<.001 ; P^{2}=0 \%$ ). All effects were accounted for by pioglitazone use. Weight gain and lower limb edema occurred more frequently with thiazolidinedione therapy (initial body weight $+2.70 \%$; $95 \% \mathrm{Cl}, 1.96 \%-4.34 \%$; $P=.001$ ). The small sample size of included RCTs prevented evaluation of more serious adverse effects of thiazolidinedione therapy.

CONCLUSIONS AND RELEVANCE Pioglitazone use improves advanced fibrosis in NASH, even in patients without diabetes. Whether this finding translates to improvement in risk for clinical outcomes requires further study.

JAMA Intern Med. 2017;177(5):633-640. doi:10.1001/jamainternmed.2016.9607 Published online February 27, 2017. Last corrected on April 4, 2017. $\leftarrow$

Invited Commentary page 640

Supplemental content
Author Affiliations: Emergency Department, Humanitas Gradenigo Hospital, Turin, Italy (Musso, Paschetta); Department of Medical Sciences, University of Turin, Turin, Italy (Cassader, Gambino).

Corresponding Author: Giovanni Musso, MD, Emergency Department, Humanitas Gradenigo Hospital, Gorso Regina Margherita 8, 10132 Turin, Italy (giovanni_musso@yahoo.it). 
$\mathrm{N}$ onalcoholic fatty liver disease (NAFLD) is the most common chronic liver disease in the world, encompassing a histological spectrum ranging from simple steatosis to steatosis plus necroinflammation, known as nonalcoholic steatohepatitis (NASH), with variable stages of fibrosis. Both fibrosis stage and NASH can only be assessed by liver biopsy. ${ }^{1}$

There is no established treatment for NASH, which is the second leading cause of liver disease among adults awaiting liver transplant and is projected by 2020 to be the leading indication for liver transplant. ${ }^{2,3}$ Extensive experimental and epidemiological evidence suggests that the presence of advanced fibrosis (stage F3-F4) (ie, bridging fibrosis or cirrhosis) on liver biopsy is the only independent predictor of poor outcomes in NAFLD; overall and liver-related mortality, liver transplant, and liver-related complications are increased in advanced fibrosis but not in patients with NASH or milder fibrosis (stage FO-F2), whose prognosis is similar to that of the general population. ${ }^{4,5}$ Recent guidelines highlight the need to identify patients with NAFLD with advanced fibrosis to target them for more intensive monitoring for the onset of complications. ${ }^{6,7}$ However, although reversal of advanced fibrosis has been generally associated with improved clinical outcomes in other causes of chronic liver disease, ${ }^{8,9}$ this stage of disease was not improved by any of the treatments evaluated to date in randomized clinical trials (RCTs) of NASH. ${ }^{10,11}$

The thiazolidinedione antidiabetic agents have been extensively evaluated in NASH. While access to rosiglitazone maleate has been restricted by the US Food and Drug Administration, pioglitazone hydrochloride continues to be recommended in current diabetes guidelines, and novel data evaluating this drug in NASH have been recently published. ${ }^{12}$ The results of a previous meta-analysis ${ }^{10}$ suggested that thiazolidinedione therapy improved histological features of NASH but not advanced fibrosis. Therefore, we analyzed the evidence on thiazolidinedione therapy in NASH, focusing on their effect in advanced fibrosis.

\section{Methods}

Data Sources and Searches

The study protocol was approved by the Humanitas Gradenigo Review Board. We searched English-language and nonEnglish-language publications in MEDLINE, Ovid MEDLINE In-Process, Cochrane Library, EMBASE, clinicaltrials.gov, PubMed, and Scopus databases from database inception through August 15, 2016. We also reviewed abstracts from annual meetings of the American Association for the Study of Liver Disease, American Gastroenterological Association, European Association for the Study of Liver, American Diabetes Association, European Association for the Study of Diabetes, and Digestive Disease Week. All included references were subjected to the same quality assessment.

Search terms were nonalcoholic steatohepatitis (NASH), nonalcoholic fatty liver disease (NAFLD), fatty liver, liver fat, steatosis, liver enzymes, transaminase, alanine aminotransferase $(A L T)$, aspartate aminotransferase (AST), $\mathrm{v}$-glutamyl trans-

\section{Key Points}

Question What is the association of thiazolidinedione therapy with advanced liver fibrosis in nonalcoholic steatohepatitis?

Findings In this meta-analysis of 8 randomized clinical trials enrolling 516 patients with biopsy-proven nonalcoholic steatohepatitis, thiazolidinedione therapy was associated with reversed advanced fibrosis, improved overall fibrosis stages, and resolution of nonalcoholic steatohepatitis. Pioglitazone hydrochloride use accounted for all of the effects of thiazolidinedione therapy in nonalcoholic steatohepatitis, and these benefits were observed in patients without diabetes as well.

Meaning Pioglitazone use improves advanced fibrosis in nonalcoholic steatohepatitis, even in patients without diabetes, and may thus halt disease progression to end-stage liver disease in this patient population.

ferase (GGT), severity of liver disease, fibrosis, advanced fibrosis, fibrosis stage F3, fibrosis stage F4, bridging fibrosis, cirrhosis, treatment, therapy, efficacy, trial, thiazolidinedione, rosiglitazone, pioglitazone, troglitazone, glitazone, and peroxisome proliferator-activated receptor $\mathrm{\gamma}$ agonist (PPAR-ү agonist). An example of the full electronic search strategy is included in the eMethods in the Supplement.

\section{Study Selection}

Inclusion criteria were English-language and non-English -language articles reporting RCTs enrolling participants of any sex or racial/ethnic origin with NAFLD or NASH, diagnosed on the basis of radiological or histological evidence of steatosis according to accepted criteria. ${ }^{1}$ Relevant meta-analyses were also included if they followed the Preferred Reporting Items for Systematic Reviews and Meta-analyses (PRISMA) guidelines. $^{13}$

Excluded from the meta-analysis were nonhuman studies, nonrandomized trials, letters, and case reports. Also excluded were studies enrolling fewer than 10 participants, articles not reporting outcomes of interest or primary data (editorials and review articles), and investigations using inadequate case definitions or enrolling patients with secondary steatosis (eg, drug-induced steatosis and total parenteral nutrition-induced steatosis).

\section{Data Extraction and Quality Assessment}

Data were extracted from each study by 2 of us (G.M. and M.C.) independently and in duplicate. Agreement between the 2 reviewers on study selection and quality assessment of studies was evaluated by к statistics, and disagreement was resolved by mutual discussion. Authors were contacted to obtain further data and to verify methodological quality. Data were then extracted from each study independently and in duplicate by 2 of us (G.M. and R.G.) using a predefined protocol and a data extraction sheet. Discrepancies were resolved by mutual discussion. Methodological quality of RCTs was assessed using each item specified by the Cochrane Risk of Bias Tool (score range, $0-8)^{14}$ (Table). Randomized clinical trials scoring higher than 6 were arbitrarily considered as having a low risk of bias. The analysis was performed in 
Table. Randomized Clinical Trials (RCTs) With Posttreatment Histological Features of the Liver Assessing Thiazolidinedione Therapy in Nonalcoholic Steatohepatitis (NASH) Included in the Meta-analysis

\begin{tabular}{|c|c|c|c|c|c|c|c|c|c|c|}
\hline Source & $\begin{array}{l}\text { No. of } \\
\text { Patients }\end{array}$ & $\begin{array}{l}\text { Mean } \\
\text { Age, y }\end{array}$ & $\begin{array}{l}\text { Male, } \\
\%\end{array}$ & $\begin{array}{l}\text { Mean } \\
\text { BMI }\end{array}$ & $\begin{array}{l}\text { Diabetes, } \\
\%\end{array}$ & $\begin{array}{l}\text { Agent } \\
\text { (Daily Dosage) }\end{array}$ & $\begin{array}{l}\text { Trial } \\
\text { Duration, mo }\end{array}$ & Comparator & $\begin{array}{l}\text { BMI } \\
\text { Change From } \\
\text { Baseline, \% }\end{array}$ & $\begin{array}{l}\text { Quality } \\
\text { Score }^{\mathrm{a}}\end{array}$ \\
\hline $\begin{array}{l}\text { Ratziu } \\
\text { et al, } 15 \\
2008\end{array}$ & 63 & 54 & 59 & 31 & 31 & $\begin{array}{l}\text { Rosiglitazone } \\
\text { maleate }(8 \mathrm{mg})\end{array}$ & 12 & Placebo & +1 & $7(\mathrm{H})$ \\
\hline $\begin{array}{l}\text { Sanyal } \\
\text { et al, } 16 \\
2004\end{array}$ & 20 & 46 & 50 & 32 & 0 & $\begin{array}{l}\text { Pioglitazone } \\
\text { hydrochloride } \\
(30 \mathrm{mg})\end{array}$ & 6 & Vitamin $\mathrm{E}$ & 0 & $7(E)$ \\
\hline $\begin{array}{l}\text { Belfort } \\
\text { et al, }{ }^{17} \\
2006\end{array}$ & 55 & 51 & 45 & 34 & 48 & $\begin{array}{l}\text { Pioglitazone } \\
\text { hydrochloride } \\
(45 \mathrm{mg})\end{array}$ & 6 & Placebo & +2.7 & $7(E)$ \\
\hline $\begin{array}{l}\text { Aithal } \\
\text { et al, } 18 \\
2008\end{array}$ & 74 & 54 & 61 & 31 & 0 & $\begin{array}{l}\text { Pioglitazone } \\
\text { hydrochloride } \\
(30 \mathrm{mg})\end{array}$ & 12 & Placebo & +3 & $7(E)$ \\
\hline $\begin{array}{l}\text { Idilman } \\
\text { et al, } 19 \\
2008\end{array}$ & 74 & 47 & 59 & 32 & 0 & $\begin{array}{l}\text { Rosiglitazone } \\
\text { maleate }(8 \mathrm{mg})\end{array}$ & 12 & $\begin{array}{l}\text { Metformin } \\
\text { hydrochloride, } \\
\text { placebo }\end{array}$ & -2.6 & $4(B, C, D, E)$ \\
\hline $\begin{array}{l}\text { Omer } \\
\text { et al, } 20 \\
2010\end{array}$ & 64 & 49 & 55 & 31 & 70 & $\begin{array}{l}\text { Rosiglitazone } \\
\text { maleate (4 mg) }\end{array}$ & 12 & $\begin{array}{l}\text { Metformin } \\
\text { hydrochloride, } \\
\text { metformin } \\
\text { hydrochloride } \\
\text { plus } \\
\text { rosiglitazone } \\
\text { maleate }\end{array}$ & 0 & $4(B, C, D, E)$ \\
\hline $\begin{array}{l}\text { Sanyal } \\
\text { et al, } 21 \\
2010\end{array}$ & 247 & 46 & 40 & 34 & 0 & $\begin{array}{l}\text { Pioglitazone } \\
\text { hydrochloride } \\
(30 \mathrm{mg})\end{array}$ & 24 & $\begin{array}{l}\text { Vitamin E, } \\
\text { placebo }\end{array}$ & +4.8 & 8 \\
\hline $\begin{array}{l}\text { Cusi } \\
\text { et al, } 12 \\
2016\end{array}$ & 101 & 51 & 70 & 34 & 51 & \multicolumn{2}{|l|}{$\begin{array}{l}\text { Pioglitazone } \\
\text { hydrochloride } \\
(45 \mathrm{mg})\end{array}$} & Placebo & +1 & 8 \\
\hline $\begin{array}{l}\text { Abbreviat } \\
\text { by height } \\
\text { a The Coc } \\
\text { with fail } \\
\text { Cochran }\end{array}$ & $\begin{array}{l}\text { : BMI, bod } \\
\text { neters squ } \\
\text { ne Risk of } \\
\text { items in pa } \\
\text { isk of Bias }\end{array}$ & $\begin{array}{l}\text { nass ind } \\
\text { ed). } \\
\text { is Tool (s } \\
\text { ntheses. } \\
\text { ol are as }\end{array}$ & $\begin{array}{l}\text { e range, } \\
\text { Iality ite } \\
\text { ows: A }\end{array}$ & $\begin{array}{l}\text { 8) score } \\
\text { of RCTs } \\
\text { equate n }\end{array}$ & $\begin{array}{l}\text { kilograms } \\
\text { RCTs is rep } \\
\text { ording to th } \\
\text { hod of sequ }\end{array}$ & & \multicolumn{4}{|c|}{$\begin{array}{l}\text { generation), B (masking of participants performed), C (masking of personnel } \\
\text { performed), D (masking of assessors performed), E (randomization } \\
\text { concealment adequate), } \mathrm{F} \text { (adequate assessment of each outcome), } \\
\mathrm{G} \text { (selective outcome reporting avoided), and } \mathrm{H} \text { (intent-to-treat analysis of the } \\
\text { results). }\end{array}$} \\
\hline
\end{tabular}

accord with the Cochrane Handbook for Systematic Reviews of Interventions ${ }^{14}$ using a statistical software program (RevMan, version 5.3.5; The Cochrane Collaboration), and data were reported according to PRISMA guidelines. ${ }^{13}$

\section{Data Synthesis and Analysis}

The primary outcome variable was a dichotomous improvement in advanced fibrosis (stage F3-F4) on liver biopsy, defined as a 2-point improvement in fibrosis stage from F3-F4 to FO-F2 on the NASH Clinical Research Network Scale. An improvement in advanced fibrosis was defined in the following 2 ways: (1) the number of individuals among all patients with NASH included in the RCT whose fibrosis stage had changed from F3-F4 to FO-F2 at the end of treatment and (2) the number of individuals among patients with NASH with advanced fibrosis (stage F3-F4) at baseline whose fibrosis stage had changed from F3-F4 to FO-F2 at the end of treatment.

Secondary dichotomous outcome variables were at least a 1-point improvement in fibrosis of any stage on the NASH Clinical Research Network Scale in patients with NASH and NASH resolution, with fibrosis stage and NASH defined according to current guidelines. ${ }^{1}$ We also evaluated adverse effects of thiazolidinedione therapy, including weight gain, lower limb edema, congestive heart failure, bone fractures, cancer, and anemia.

Dichotomous variables are presented as odds ratios (ORs) with $95 \%$ CIs, and continuous variables are presented as weighted mean differences with $95 \%$ CIs. We conservatively used randomeffects models, with significance set at $P=.05$. Statistical heterogeneity was assessed with the $I^{2}$ statistic. Using $I^{2}$ of $50 \%$ or higher, we planned to explore individual study characteristics and those of subgroups in the main body of evidence.

Sensitivity analysis was performed by removing 1 study at a time and repeating the meta-analysis to assess whether any single study substantially affected pooled estimates. In addition, we planned a priori subgroup analysis according to the following criteria: RCTs evaluating rosiglitazone use vs RCTs evaluating pioglitazone use, RCTs enrolling exclusively patients without diabetes vs RCTs also enrolling patients with diabetes, and treatment duration of 1 year or less vs longer than 1 year and different dosages, as well as for each item of the Cochrane Risk of Bias Tool.

When at least 8 comparisons were available, the effect of age, changes in insulin resistance (as estimated by the homeostasis model of insulin resistance index), and treatment duration on assessed outcomes was evaluated by meta-regression analysis (using random-effects models, with within-study variance estimated with the unrestricted maximum likelihood method). Publication bias was examined using funnel plots and the Egger test.

\section{Management of Missing Data}

Missing data were managed by contacting the corresponding authors of the RCTs. If this contact was unsuccessful, missing 


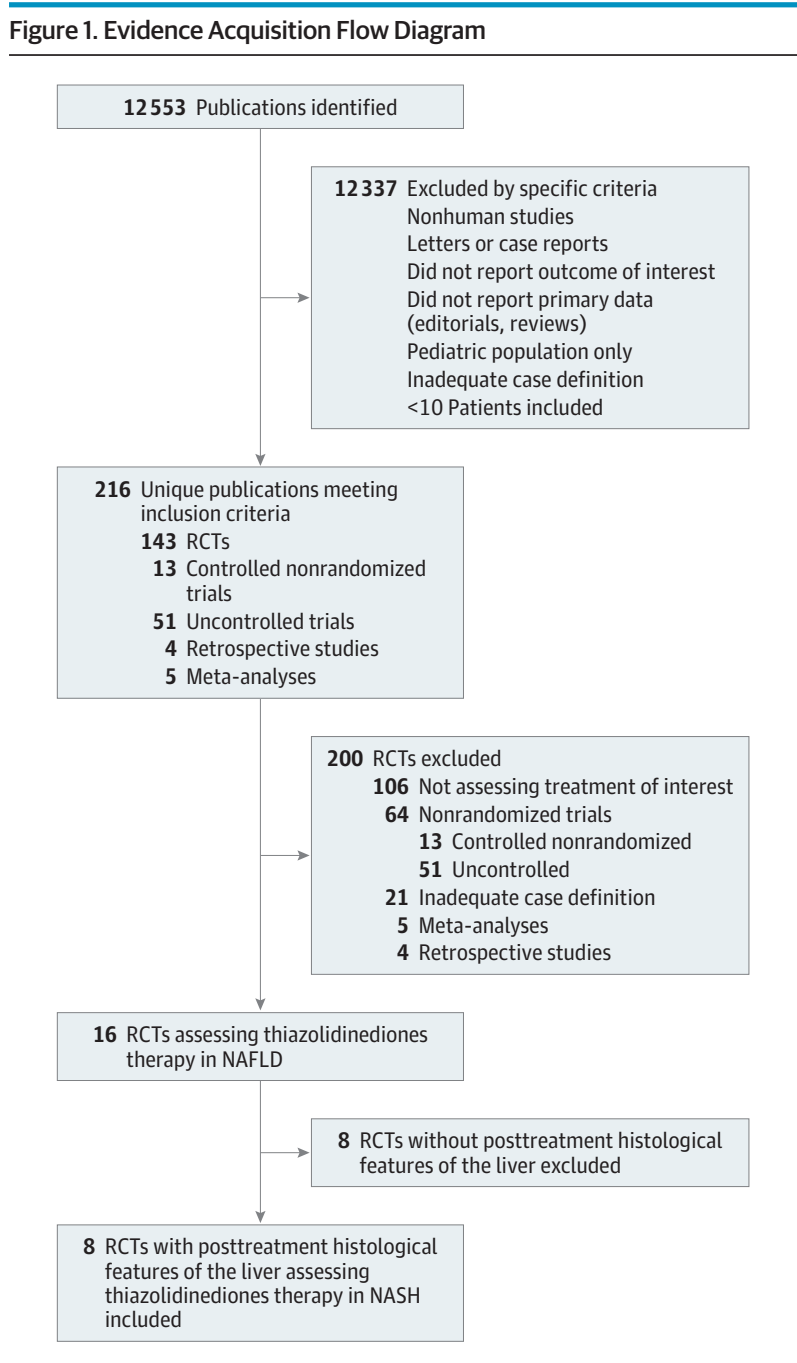

NAFLD indicates nonalcoholic fatty liver disease; NASH, nonalcoholic steatohepatitis; and RCTs, randomized clinical trials.

histological scores were calculated from the raw numbers given in tables or estimated from bar charts. For missing SDs of the mean change in scores and where the $P$ value was provided for a comparison between treated and control groups, the SD was calculated by converting the $P$ value to a $t$ statistic with appropriate $d f$ and then calculating SEs and SDs. If neither the SD nor the $P$ values were supplied, imputation of an SD from studies with similar measurement methods, trial duration, and measurement error was used if available, tested in a sensitivity analysis, and reported if the estimate differed meaningfully from previous estimates.

\section{Results}

The agreement between reviewers was good to excellent. The к statistics were 0.88 for study selection and 0.92 for quality assessment.

A flow diagram of study selection is shown in Figure 1. We identified 8 RCTs ( 5 evaluating pioglitazone use and 3 evaluating rosiglitazone use, with posttreatment histological features of the liver) enrolling 516 patients. Trial durations were 6 to 24 months, with daily dosages ranging from 4 to 8 $\mathrm{mg}$ for rosiglitazone maleate and from 30 to $45 \mathrm{mg}$ for pioglitazone hydrochloride (Table). ${ }^{12,15-21}$ For the included RCTs, the histopathological scoring system proposed by the NASH Clinical Research Network was used to score the severity of histological features of the liver, as recommended by current guidelines. ${ }^{1,6,7}$ Fibrosis was assessed by Masson trichrome stain, and the pathologist intraobserver and interobserver agreement for fibrosis staging was good to excellent ( $\mathrm{\kappa}$ statistic, $\geq 0.82$ ).

The agreement between reviewers for quality assessment was good ( $\mathrm{k}$ statistic, 0.84). Overall, 6 RCTs had a low risk of bias in key domains, while 2 RCTs (both evaluating rosiglitazone use) demonstrated a higher risk of bias because of unclear blinding and randomization concealment. ${ }^{19,20}$

Pooled results of RCTs showed that thiazolidinedione therapy was associated with improved advanced fibrosis (Figure 2). The effect size was significant when considering all patients with NASH (Figure 2A) and only patients with NASH with advanced fibrosis at baseline (Figure 2B). In addition, thiazolidinedione therapy was associated with improved fibrosis of any stage and induced NASH resolution (Figure 3). Statistical heterogeneity was low for all evaluated outcomes, suggesting a consistent effect size across studies.

After the analysis to RCTs enrolling exclusively to patients without diabetes was restricted, pooled ORs remained similar in magnitude and direction to the overall effect. Among the 4 studies, thiazolidinedione therapy was associated with improvement in advanced fibrosis (OR, 2.95; 95\% CI, 1.04-10.90; $\left.P=.02 ; I^{2}=0 \%\right)$, improvement in fibrosis of any stage (OR, 1.76; 95\% CI, 1.02-3.03; $P=.02$; $I^{2}=0 \%$ ), and NASH resolution (OR, 3.40; 95\% CI, 1.95-5.93; $\left.P<.001 ; I^{2}=0 \%\right)$.

Trial duration, dosage, and exclusion of the 2 RCTs ${ }^{16,18}$ with a high risk of bias (both evaluating rosiglitazone use) did not affect the magnitude and direction of the overall effect. Metaregression analysis found no association between assessed outcomes and age, homeostasis model of insulin resistance index, and treatment duration.

The separate analyses of rosiglitazone and pioglitazone demonstrated that the observed effects of thiazolidinedione therapy were accounted for by pioglitazone use. Rosiglitazone use did not reach statistical significance for any histological outcome (Figure 2).

The Egger test and funnel plot analysis found no strong evidence for publication bias. These results are shown in eFigure 1 in the Supplement.

Thiazolidinedione therapy was associated with a mean $2.7 \%$ weight gain compared with controls (eFigure 2 in the Supplement). It was also associated with a higher OR for lower limb edema $\left(2.36\right.$; 95\% CI, 1.15-4.84; $\left.P=.02 ; I^{2}=0 \%\right)(6$ studies), without any significant difference in agents, RCTs, or trial duration.

Reporting of other adverse events was variable. Recognized adverse effects, such as congestive heart failure, were reported in fewer than half of the RCTs. 
Figure 2. Thiazolidinedione Therapy (TZD) and Improvement in Advanced Fibrosis, Improved Fibrosis of Any Stage, and Nonalcoholic Steatohepatitis (NASH) Resolution

A All patients with NASH

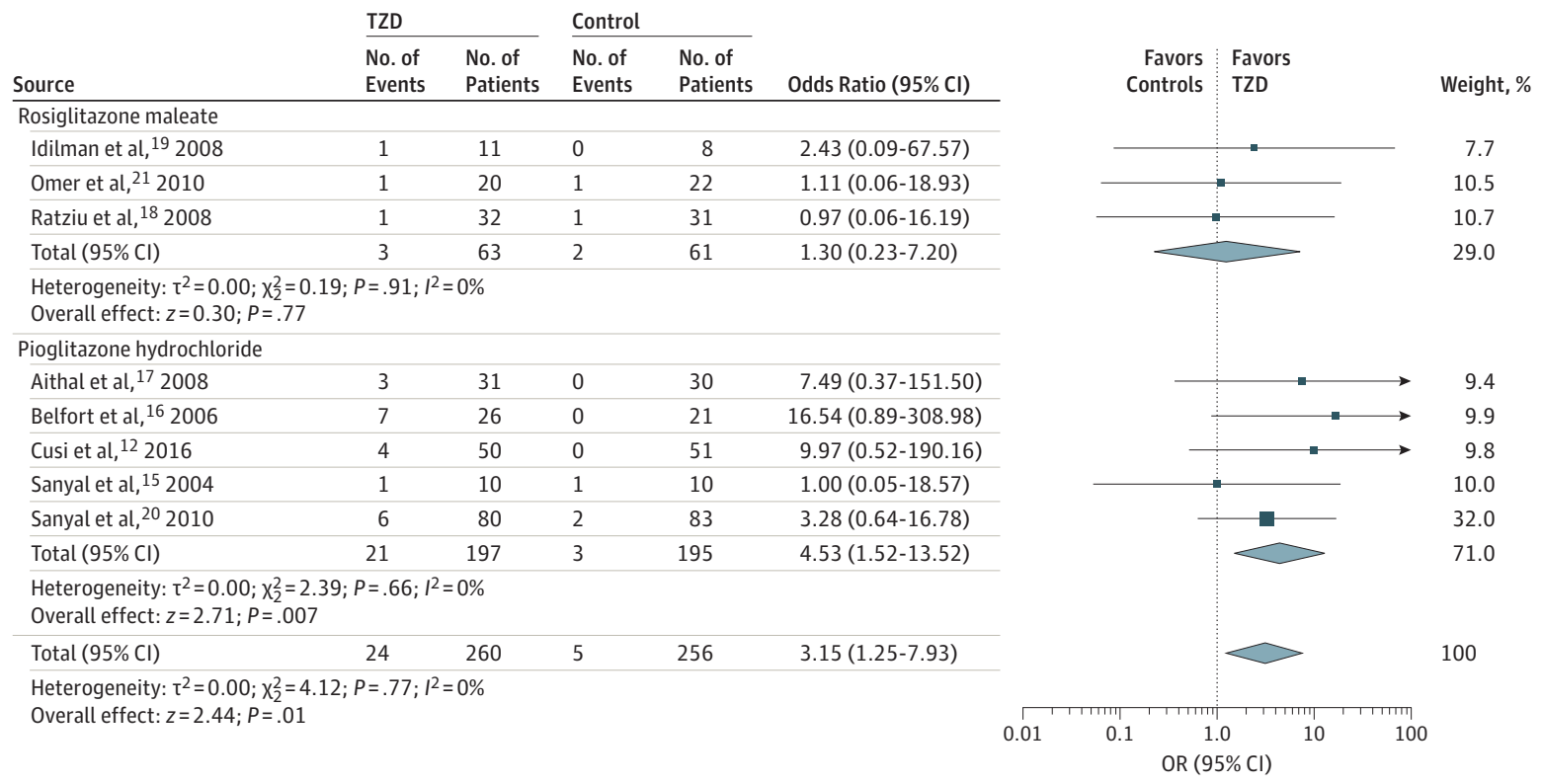

B Patients with NASH with advanced fibrosis at baseline

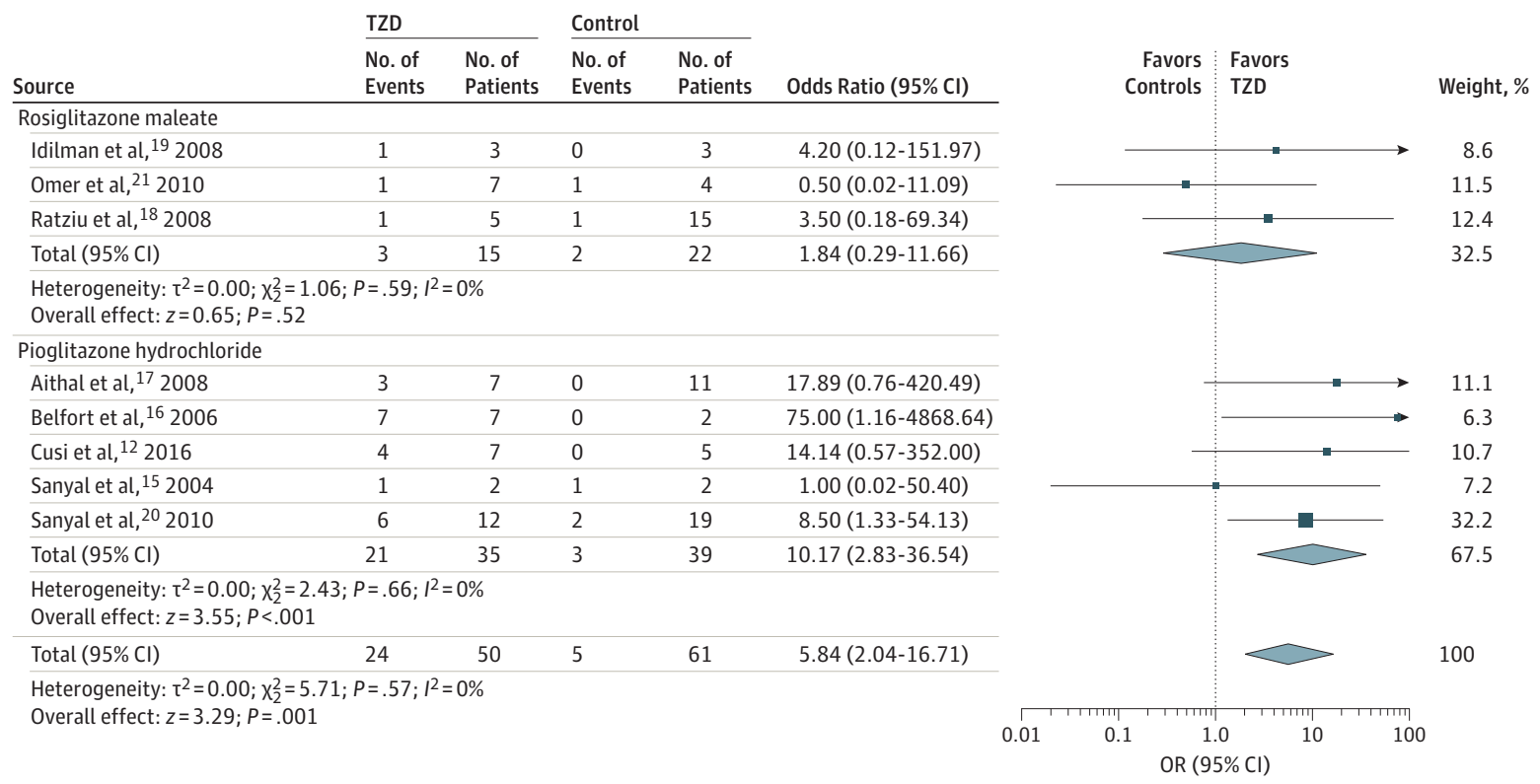

A, Improvement in advanced fibrosis (stage $\mathrm{F} 3-\mathrm{F} 4$ ) in patients with biopsy-proven NASH, defined as the number of patients with NASH whose fibrosis stage had changed from F3-F4 to FO-F2 at the end of treatment. $\mathrm{B}$, Improvement in advanced fibrosis (stage $\mathrm{F} 3-\mathrm{F} 4$ ) in patients with NASH with advanced fibrosis, defined as the number of patients with NASH with advanced (F3-F4) fibrosis at baseline whose fibrosis stage had changed from F3-F4 to FO-F2 at the end of treatment. In contrast to A, only patients with NASH and advanced fibrosis were included as the denominator in $\mathrm{B}$.

\section{Discussion}

In this meta-analysis of 8 RCTs of thiazolidinedione therapy, we found that treatment for up to 24 months was associated with improved advanced fibrosis and fibrosis of any stage and
NASH resolution. These effects were mainly accounted for by pioglitazone use. Benefits were also observed in patients with NASH without diabetes. Aside from weight gain and lower limb edema, no major adverse events were reported during the trial durations, with recognizable power limitations of our analysis because of the few included RCTs. 
Figure 3. Improved Fibrosis of Any Stage and Nonalcoholic Steatohepatitis (NASH) Resolution

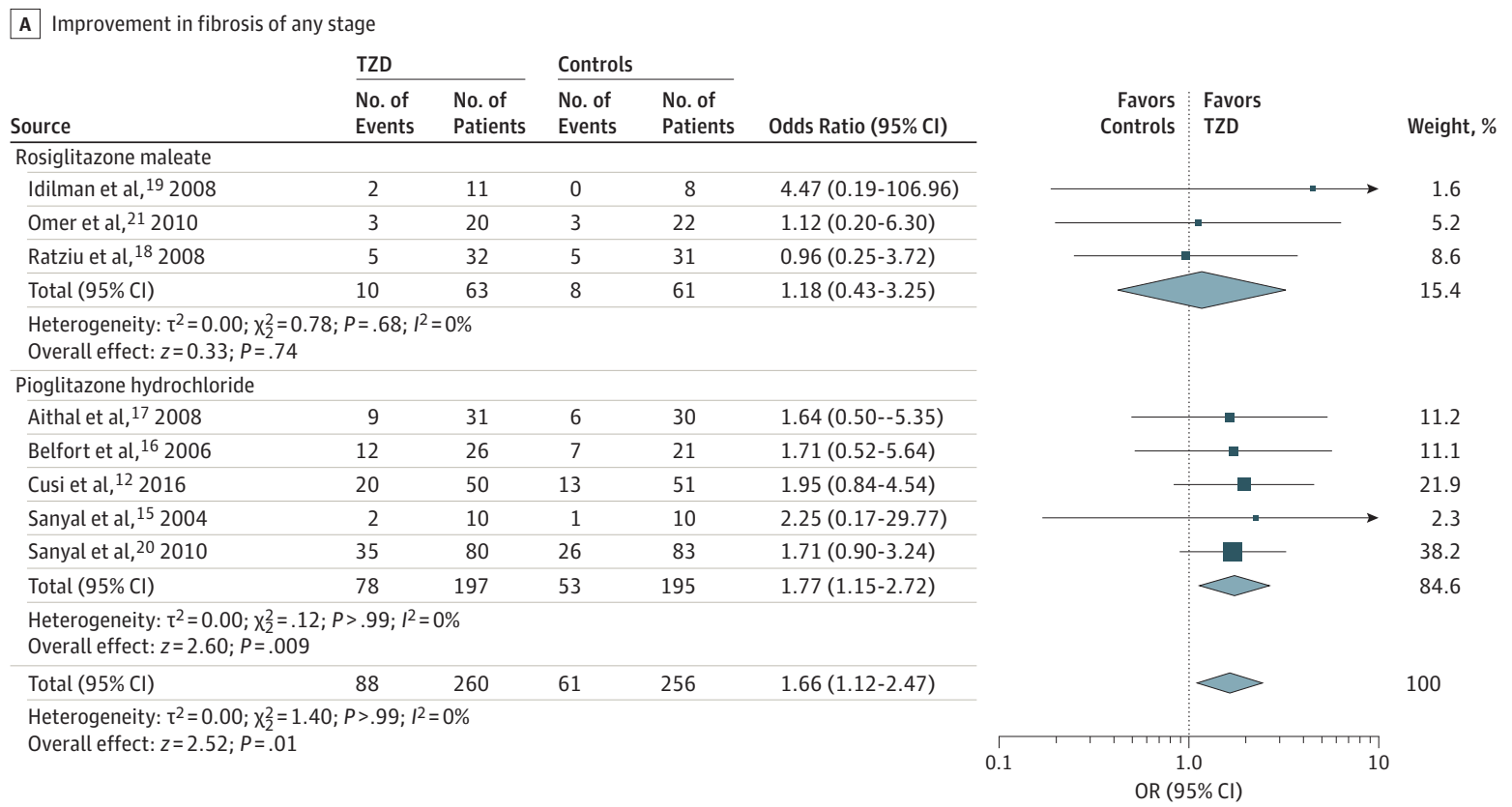

B Induction of NASH resolution

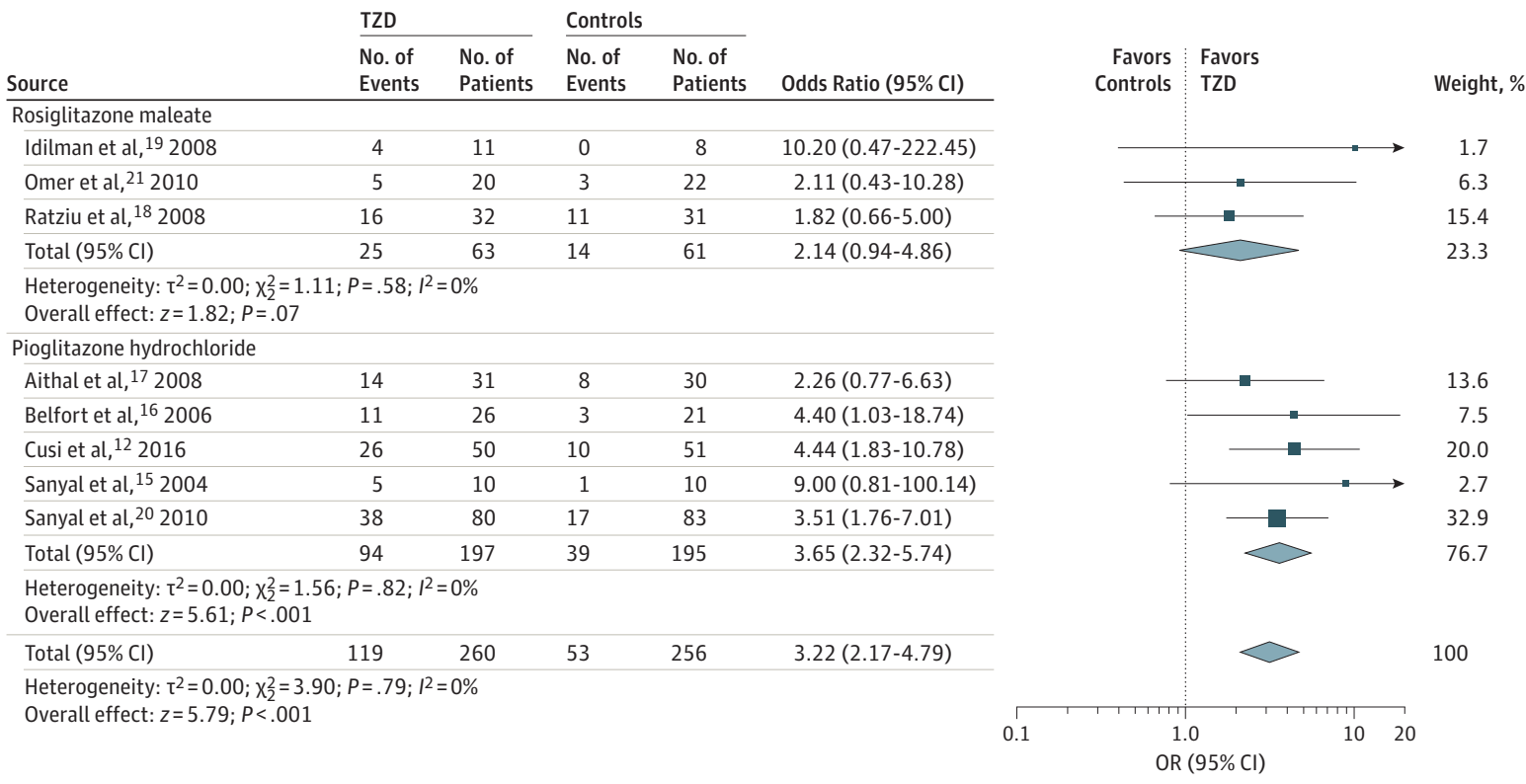

A, Improvement by at least 1 stage in liver fibrosis of any stage in patients with biopsy-proven NASH. B, Effects of thiazolidinedione therapy on NASH resolution, defined as the number of patients with NASH who showed NASH resolution at the end of treatment. OR indicates odds ratio.
Nonalcoholic steatohepatitis is becoming a major public health issue and is a leading cause of liver transplant. ${ }^{2,3,6,7,22}$ Among the clinical and histological features of NAFLD, the severity of liver fibrosis has been mechanistically and epidemiologically linked to increased overall and liver-related mortality and liver-related complications. Specifically, advanced fibrosis (stage F3-F4) (ie, bridging fibrosis or cirrhosis) on liver biopsy is the strongest independent predictor of poor outcomes in NAFLD and NASH and has been recognized in re- cent guidelines as the main diagnostic and therapeutic target to halt NASH progression to end-stage liver disease, change the natural history of the disease, and improve long-term prognosis of patients with NASH. ${ }^{4-7,23,24}$ Unfortunately, none of the pharmacological agents evaluated in prior phase 1,2 , and 3 randomized trials showed improvement of advanced fibrosis in patients with NASH. ${ }^{10,11}$

We found that thiazolidinedione therapy (specifically pioglitazone use) for up to 24 months was associated with a 
reversal of advanced fibrosis stage in NASH and may thus improve long-term prognosis in this subgroup of patients who are at higher risk of poor liver-related outcomes. ${ }^{4-7}$ These benefits were also observed in patients without diabetes, which may prompt the extension of approved indications for pioglitazone use.

It is unclear why pioglitazone use (and not rosiglitazone use) accounted for all of the benefits observed with thiazolidinedione therapy in our analysis, indicating that this observation may not be a class effect of these drugs. Possible differences can be explained by the differential effects of pioglitazone and rosiglitazone on inflammation and fibrosis mechanisms, such as through up-regulation of adiponectin, activation of adenosine monophosphate-activated protein kinase, and induction of hepatic stellate cell senescence. ${ }^{25,26}$

The benefits of pioglitazone use should be weighed against its adverse effects, which in our analysis were limited to weight gain and lower limb edema. The short trial durations $(\leq 24$ months) and few included RCTs may have limited the power of our analysis to detect more serious adverse effects of thiazolidinedione therapy. Therefore, careful tailoring of individual risk-benefit profiles will be essential to limit exposure to adverse effects of pioglitazone use. The restriction of pioglitazone use to those patients with advanced liver fibrosis and a higher risk of liver-related death seems plausible. Two patients with NASH with advanced fibrosis would be needed to take pioglitazone to improve advanced fibrosis in 1 patient (number needed to treat, 1.9).

Limiting the duration of pioglitazone use may also reduce exposure to adverse effects. Within this context, 2 trials ${ }^{12,27}$ suggest that the use of thiazolidinedione therapy beyond 18 months does not offer significant additional histological benefit. Finally, a key issue after pioglitazone discontinuation is the prevention of liver disease recurrence. Other study $^{28}$ results suggest that the durability of histological response obtained with thiazolidinedione therapy depends on the achievement of sustained lifestyle changes, particularly increased physical activity.

\section{Limitations}

Limitations of our meta-analysis, which are inherent to the nature of the included studies, need to be mentioned. Included RCTs had small sample sizes and evaluated the effect of thiazolidinedione therapy on histological features of the liver rather than on clinical outcomes. However, as acknowledged by consensus of the American Association for the Study of Liver Diseases and the US Food and Drug Administration, ${ }^{29}$ because of the slow progressive nature of NASH it is impractical and unfeasible to perform larger studies of long duration to identify treatment-related clinical benefits, and histological features of the liver offer the best surrogate measure of the risk of liverrelated complications. Among all histological features of NAFLD, advanced liver fibrosis has been mechanistically and epidemiologically linked to an increased risk of adverse liverrelated complications in NAFLD, and reversal of advanced fibrosis portends an improved prognosis in diverse causes of chronic liver disease..$^{8,9,11}$

\section{Conclusions}

Recent guidelines recommend identification of patients with NAFLD with advanced fibrosis to target them for more intensive monitoring of the onset of complications but acknowledge the lack of therapeutic options that effectively reverse advanced stages of liver disease. ${ }^{1,6,7}$ The new finding in this meta-analysis is that treatment with the antidiabetic drug pioglitazone reverses the more advanced stages of liver disease in NASH regardless of the presence of diabetes, which provides a rationale for evaluating the effect of this drug on clinical outcomes in this subgroup of patients at higher risk of liverrelated complications.

\section{ARTICLE INFORMATION}

Accepted for Publication: November 27, 2016.

Correction: This article was corrected on April 4, 2017, to fix headings over forest plots of Figures 2 and 3.

Published Online: February 27, 2017. doi:10.1001/jamainternmed.2016.9607

Author Contributions: Dr Musso had full access to all of the data in the study and takes responsibility for the integrity of the data and the accuracy of the data analysis.

Study concept and design: Musso.

Acquisition, analysis, or interpretation of data:

Musso, Paschetta, Gambino.

Drafting of the manuscript: Musso.

Critical revision of the manuscript for important intellectual content: Musso, Paschetta, Gambino. Statistical analysis: Musso, Paschetta.

Administrative, technical, or material support: Musso.

Study supervision: Musso, Gambino.

Conflict of Interest Disclosures: None reported.

\section{REFERENCES}

1. Chalasani N, Younossi Z, Lavine JE, et al. The diagnosis and management of non-alcoholic fatty liver disease: practice guideline by the American Association for the Study of Liver Diseases, American College of Gastroenterology, and the American Gastroenterological Association. Hepatology. 2012;55:2005-2023.

2. Wong RJ, Aguilar M, Cheung R, et al. Nonalcoholic steatohepatitis is the second leading etiology of liver disease among adults awaiting liver transplantation in the United States. Gastroenterology. 2015;148(3):547-555.

3. Singal AK, Guturu P, Hmoud B, Kuo YF, Salameh $\mathrm{H}$, Wiesner RH. Evolving frequency and outcomes of liver transplantation based on etiology of liver disease. Transplantation. 2013;95(5):755-760.

4. Ekstedt $M$, Hagström $H$, Nasr $P$, et al. Fibrosis stage is the strongest predictor for disease-specific mortality in NAFLD after up to 33 years of follow-up. Hepatology. 2015;61(5):1547-1554.

5. Angulo $P$, Kleiner DE, Dam-Larsen $S$, et al. Liver fibrosis, but no other histologic features, is associated with long-term outcomes of patients with nonalcoholic fatty liver disease. Gastroenterology. 2015;149(2):389-97.e10. doi:10.1053/j.gastro.2015.04.043

6. European Association for the Study of the Liver (EASL); European Association for the Study of Diabetes (EASD); European Association for the Study of Obesity (EASO). EASL-EASD-EASO Clinical Practice Guidelines for the management of non-alcoholic fatty liver disease. Diabetologia. 2016;59(6):1121-1140.

7. National Institute for Health and Care Excellence (NICE). Guideline: non-alcoholic fatty liver disease (NAFLD): assessment and management. http://www.nice.org.uk/guidance/ng49?unlid= 311500465201610282942 . Published July 2016. Accessed August 10, 2016.

8. Mallet V, Gilgenkrantz H, Serpaggi J, et al. Brief communication: the relationship of regression of cirrhosis to outcome in chronic hepatitis C. Ann Intern Med. 2008;149(6):399-403.

9. Rockey DC. Liver fibrosis reversion after suppression of hepatitis B virus. Clin Liver Dis. 2016; 20(4):667-679. 
10. Musso G, Cassader M, Rosina F, Gambino R. Impact of current treatments on liver disease, glucose metabolism and cardiovascular risk in non-alcoholic fatty liver disease (NAFLD): a systematic review and meta-analysis of randomised trials. Diabetologia. 2012;55(4):885-904.

11. Musso G, Cassader M, Gambino R. Non-alcoholic steatohepatitis: emerging molecular targets and therapeutic strategies. Nat Rev Drug Discov. 2016;15 (4):249-274.

12. Cusi K, Orsak B, Bril F, et al. Long-term pioglitazone treatment for patients with nonalcoholic steatohepatitis and prediabetes or type 2 diabetes mellitus: a randomized trial. Ann Intern Med. 2016;165(5):305-315.

13. Moher D, Liberati A, Tetzlaff J, Altman DG; PRISMA Group. Preferred Reporting Items for Systematic Reviews and Meta-analyses: the PRISMA statement. J Clin Epidemiol. 2009;62(10): 1006-1012.

14. Higgins JPT, Green S, eds. Cochrane Handbook for Systematic Reviews of Interventions: Version 5.1.0. http://handbook.cochrane.org/. Updated March 2011. Accessed July 14, 2016.

15. Ratziu V, Giral P, Jacqueminet $S$, et al; LIDO Study Group. Rosiglitazone for nonalcoholic steatohepatitis: one-year results of the randomized placebo-controlled Fatty Liver Improvement With Rosiglitazone Therapy (FLIRT) trial. Gastroenterology. 2008;135(1):100-110.

16. Sanyal AJ, Mofrad PS, Contos MJ, et al. A pilot study of vitamin $E$ versus vitamin $E$ and pioglitazone for the treatment of nonalcoholic steatohepatitis. Clin Gastroenterol Hepatol. 2004;2 (12):1107-1115
17. Belfort R, Harrison SA, Brown $K$, et al. A placebo-controlled trial of pioglitazone in subjects with nonalcoholic steatohepatitis. N Engl J Med. 2006;355(22):2297-2307.

18. Aithal GP, Thomas JA, Kaye PV, et al. Randomized, placebo-controlled trial of pioglitazone in nondiabetic subjects with nonalcoholic steatohepatitis. Gastroenterology. 2008;135(4):1176-1184

19. Idilman R, Mizrak D, Corapcioglu D, et al. Clinical trial: insulin-sensitizing agents may reduce consequences of insulin resistance in individuals with non-alcoholic steatohepatitis. Aliment Pharmacol Ther. 2008;28(2):200-208.

20. Omer Z, Cetinkalp S, Akyildiz M, et al. Efficacy of insulin-sensitizing agents in nonalcoholic fatty liver disease. Eur J Gastroenterol Hepatol. 2010;22 (1):18-23.

21. Sanyal AJ, Chalasani N, Kowdley KV, et al; NASH CRN. Pioglitazone, vitamin E, or placebo for nonalcoholic steatohepatitis. N Engl J Med. 2010; 362(18):1675-1685

22. Musso G, Gambino R, Tabibian JH, et al. Association of non-alcoholic fatty liver disease with chronic kidney disease: a systematic review and meta-analysis. PLoS Med. 2014;11(7):e1001680. doi:10.1371/journal.pmed.1001680

23. Musso G, Gambino R, Cassader M, Pagano G. Meta-analysis: natural history of non-alcoholic fatty liver disease (NAFLD) and diagnostic accuracy of non-invasive tests for liver disease severity. Ann Med. 2011;43(8):617-649.

24. Musso G, Cassader M, Cohney S, et al. Fatty liver and chronic kidney disease: novel mechanistic insights and therapeutic opportunities. Diabetes Care. 2016;39(10):1830-1845.

25. Musso G, De Michieli F, Bongiovanni D, et al. New pharmacologic agents that target inflammation and fibrosis in nonalcoholic steatohepatitis-related kidney disease [published online August 10, 2016]. Clin Gastroenterol Hepatol. doi:10.1016/j.cgh.2016.08.002

26. Panebianco $C$, Oben JA, Vinciguerra $M$, Pazienza V. Senescence in hepatic stellate cells as a mechanism of liver fibrosis reversal: a putative synergy between retinoic acid and PPAR-gamma signaling [published online September 21, 2016] Clin Exp Med. doi:10.1007/s10238-016-0438-x

27. Ratziu V, Charlotte F, Bernhardt C, et al; LIDO Study Group. Long-term efficacy of rosiglitazone in nonalcoholic steatohepatitis: results of the fatty liver improvement by rosiglitazone therapy (FLIRT 2) extension trial. Hepatology. 2010;51(2):445-453. 28. Argo CK, lezzoni JC, Al-Osaimi AM, Caldwell $\mathrm{SH}$. Thiazolidinediones for the treatment in $\mathrm{NASH}$ : sustained benefit after drug discontinuation? J Clin Gastroenterol. 2009;43(6):565-568.

29. Sanyal AJ, Friedman SL, McCullough AJ, Dimick-Santos L; American Association for the Study of Liver Diseases; United States Food and Drug Administration; Food and Drug Administration Joint Workshop. Challenges and opportunities in drug and biomarker development for nonalcoholic steatohepatitis: findings and recommendations from an American Association for the Study of Liver Diseases (AASLD)-Food and Drug Administration (FDA) joint workshop. Hepatology. 2015;61(4): 1392-1405.

\section{The Role of Pioglitazone in the Management of Nonalcoholic Steatohepatitis Are We There Yet?}

Hal F. Yee Jr, MD, PhD

Nonalcoholic steatohepatitis (NASH) and its clinical sequelae have become an increasingly prevalent and important cause of hepatic morbidity and mortality. ${ }^{1}$ Despite almost 2 decades of intense study, we are still not certain how best to treat NASH. In this issue of JAMA Internal Medicine, Musso and colleagues present a meta-analysis that suggests that thizaolidinedione use is associatyed with improvement in advanced fibrosis in NASH, even in Related article page 633 patients without diabetes. ${ }^{2}$ Other medications in the thiazolidinedione class did not demonstrate a significant effect in treatment of nonalcoholic steatohepatitis, therefore this commentary focuses on pioglitazone. Although this article makes a significant contribution to our understanding of the potential role that this thiazolidinedione may have in the management of NASH, there remain substantive questions that need to be addressed before pioglita- zone hydrochloride can be recommended as a treatment for patients with NASH.

Does pioglitazone alter clinical outcomes, such as development of ascites or encephalopathy, need for liver transplantation, or liver-related death? This meta-analysis identified significant improvements in histological features of the liver in patients treated with pioglitazone, but it did not look at clinical outcomes. Although liver histological status is a commonly used surrogate outcome in evaluating the efficacy of treatments for other hepatic conditions, it might not correlate as well with clinical outcomes in patients with NASH, who are often obese and have type 2 diabetes and other complications of metabolic syndrome. Such patients are at high risk for serious cardiovascular and neurovascular complications (ie, myocardial infarction or stroke), which could influence clinical outcome more strongly than the complications of NASH. Further longitudinal studies will be required to determine if pioglitazone improves clinical outcomes. 